\title{
The effect of PV siting on power system flexibility needs.
}

\author{
Michaelangelo Tabone, Christoph Goebel, and Duncan Callaway
}

September 24, 2016

\begin{abstract}
Locations of photovoltaic (PV) systems affect the variability and uncertainty of their power generation, and as a result the amount of flexible resources needed to balance supply and demand. However, studies on the integration of renewable electricity into power systems focus on the total amount of renewable generation, and not their locations. This paper uses a hidden state, spatial-statistical model to simulate how locational arrangements and balancing policies affect the need for reserves - load following and regulation - in California's power system when including $12 \mathrm{GW}$ of photovoltaic generators and $9.5 \mathrm{GW}$ of wind.

Our results show that locations of utility-scale PV systems significantly affect on the amount of reserves needed for balancing their variability and uncertainty. When PV is geographically dispersed the additional load following and regulation reserve needs are small; on average $<1.2 \%$ and $<0.05 \%$ of installed PV capacity respectively. These rise to $5.6 \%$ and $0.2 \%$ in centralized scenarios. Most the benefits of this dispersion can be achieved with relatively few, 25, systems. These are sized at roughly $500 \mathrm{MW}$ each, which is about the size of the largest systems in California today. Almost all of the load following reserve need is driven by errors in the hourly forecasts of solar generation. These can be mitigated either by better forecasts, or dispersing plants.

Siting policies for PV must weigh system flexibility against other locational concerns, such as the energy and capacity value of the solar resource in an area. We find a small trade off between energy and reserves; where dispersed systems require less reserves but also have lower capacity factors than more centralized systems. However, we find a much greater trade-off between energy and capacity value in California; where the regions that produce the most energy on average - in the Mojave desert - tend to be cloudy during current peak demand hours, which occur during Summer afternoons.
\end{abstract}

\section{Introduction}

Renewable generation poses a dual challenge to electricity system planners and operators. It adds variability and uncertainty in electricity supply, while decreasing the number of on-line controllable generators that can be used to balance supply and demand. For electric power systems to rely primarily on renewable generation, flexible resources will be needed. These resources could be generators, consumers, or storage systems that can reliably increase or decrease supply or demand to maintain balance.

A number of renewables integration studies [1-7] investigate how increasing the amount of wind and solar generation will impact power system operations. Among other things, these studies estimate the amount of flexible resources needed to be held in reserve to respond to fast 
or unexpected changes in supply or demand. Notably, Wu et al. find that including the PV capacity amounting to $20 \%$ of peak demand in the Arizona Public Service territory results in increases in reserve needs by $2 \%$ to $3 \%$ of installed PV capacity [1].

Locations of wind and solar generators affect the need for flexibility. Renewable resources exhibit different amounts of variability and uncertainty regionally, and often renewable resources are spatially correlated. Spatial correlation implies that renewable generators located closer together are more likely to experience simultaneous weather events than those far apart. Thus siting PV systems in close proximity to one another may exacerbate events that need to be managed by the system operator, and the opposite may be true for systems sited far apart from one another.

Renewables integration studies often account for spatial correlation of PV generation; however deciding where to site new PV or transmission is not their focus. For example, Phase one of the Western Wind and Solar Integration Study uses spatial scenarios to vary how much PV is sited by state. But within each state, PV is always sited according to the same principles [4,5]. States are large enough that moving systems from one to another does not greatly affect the correlation between systems, but the spacing of systems within states will affect this correlation.

In this study we seek to answer questions about how siting PV will affect reserve requirements. For example: What are the implications of siting large amounts of PV at the same substation, or in the same county? Can utility-scale systems achieve the same benefits of spatial diversity that rooftop systems do? Do regions with low variability and uncertainty overlap with regions with high capacity factor, or with high capacity value? To our knowledge these questions have not been directly and systematically addressed in the literature. The answers to these types of questions could be important for regional transmission planning processes, such as those in California [8].

\subsection{Statistics of solar variability and uncertainty}

A large body of prior work focuses on the spatial correlation of variability from wind and solar generation. See [9-12] for examples regarding solar with complete literature reviews, and $[13,14]$ for examples regarding wind. Most of these studies use spatial correlation functions to predict the standard deviation of variability or uncertainty from theoretical arrangements of PV systems $[12,15,16]$, and do so accurately.

Despite knowledge of the standard deviation, it remains difficult to predict variability or uncertainty from PV in a way that is useful for power system operations or planning. System operators are concerned with high impact, low probability events which are defined by distribution tails - for example, system operators may plan to balance supply and demand in $>95 \%$ of times. Mean and standard deviation completely parametrize only a few distribution shapes, most notably the Gaussian distribution. Unfortunately, the distribution of variability from PV has been shown not to be Gaussian in many studies $[10,12,17,18]$.

Two studies condition variability on hidden Markov processes to predict non-Gaussian shapes $[2,19]$. However these models predict variability and not uncertainty in PV generation, and do so only for individual systems, without modeling spatial correlation of neighboring systems. Another strategy accounts for spatial diversity by smoothing the generation from a small PV system-or a single irradiance point sensor-to mimic generation from a larger plant [18, 20,21]. Different smoothing filters are applied at different time-scales to represent increased spatial correlation at longer timescales and vice versa. While this method accounts for smoothing in 
one utility-scale PV system, it does not model spatial correlation between multiple utility-scale systems, which is precisely what we would like to study in this paper.

Finally, the literature tends to focus on predicting statistics, and not on predicting the resulting effect on power system operations and planning. Two notable exceptions investigate the costs of mitigating ramps in solar generation. Mills and Wiser investigate the costs of managing changes in solar generation ramps when dispersing PV systems from 1 to 25 locations [10], though this study focuses only on variability and not uncertainty, and does not account for intra-plant smoothing. Perez et al. investigate the costs of using electricity storage to maintain ramps in PV generation below a specified threshold [22], but do not investigate the effect of these ramps on power system operations.

We advance this body of work by applying modeling techniques from our prior work [23], in which we developed a statistical model that addresses these issues by (a) accounting for spatial correlation, (b) predicting metrics of variability and uncertainty that are directly relevant to grid operation and planning, (c) and predicting boundaries on distribution tails that are consistent with observed data. This paper makes use of this model to estimate how the spatial arrangement of PV in California may affect the need for reserves.

\subsection{Predicting reserve need in renewable integration studies}

Renewable integration studies treat reserve procurement in differing ways - driven both by the procedures of system operators and by the assumptions made in each study. For example, many studies define the amount of generation held in reserve to be constant throughout the year, or to be a fixed percentage of total load, wind, and/or solar in the system [5,24]. In contrast, more recent studies define the amount of reserves procured each hour to be dynamic, reflecting varying expectations of load and renewable generation in each specific hour [1-3].

Methods to calculate the amount of reserves to be procured - dynamic or constant - also vary. Most early studies used the $n$-sigma method, which defines reserve need to be $n$ standard deviations of expected variability and/or uncertainty. If distribution shape of variability or uncertainty is Gaussian, the n-sigma method defines a confidence interval for reserve needs. However the distribution shape has been shown not to be Gaussian. It has heavier tails, signifying that extreme events are more likely than a Gaussian would predict $[25,26]$. Integration studies will often use an arbitrarily large $n$ as a conservative approximation for boundaries on these wide tails.

More recent studies overcome this limitation by using alternative heuristics. For example, the 2nd Phase of the Western Wind and Solar Integration Study define regulation reserve procurement by the the square root of the sum of squares of the $95^{t h}$ percentile hourly forecast errors for wind and solar [4]. Wu et al. actually simulate 200 possibilities for reserve need at each interval, and procure reserves that will cover $95 \%$ of simulated need [1].

\subsection{Overview}

In this study, we build on our earlier modeling work and upon previous renewable integration studies to estimate how locations of PV systems affect the need for reserves in future power systems. We use California's long-term planning process as a case study, modeling the need for reserves when load, wind and solar are fixed at levels that are expected to meet the state's $33 \%$ by 2020 goals: $12 \mathrm{GW}$ of photovoltaics and $9.5 \mathrm{GW}$ of wind [27]. We then vary the locations of PV 
systems to compare centralized versus distributed arrangements, centralized versus distributed balancing authorities, and regional climates. In doing so, we answer the question of how much geographic dispersion of PV systems can mitigate flexibility need in a realistic future power systems.

In addition to quantifying reserve needs, we also estimate of the energy production and capacity value of solar generation in these different scenarios. In doing so we explore trade-offs between siting PV systems to minimize reserves, or to maximize energy production or capacity value.

\section{Method}

\section{$2.1 \quad$ Locational scenarios}

Table 1 lists each of the locational scenarios we examine in this paper; each includes $12 \mathrm{GW}$ of rated PV capacity. ${ }^{1}$ Six scenarios represent arrangements of utility-scale PV plants ranging from highly concentrated to highly dispersed. ${ }^{2}$ We limited candidate locations to 130 "ecologically preferred" regions, which were randomly selected from all possible locations outside of the environmentally excluded zones identified in $[28] .{ }^{3}$ In the first five utility scale scenarios we site plants where they are expected to have the maximal capacity factor (see Figure 1), i.e. where they have the greatest expected energy production. For the sixth utility-scale scenario we site a single $12 \mathrm{GW}$ system where we expect it to have the greatest capacity value, i.e. the site with the maximal expected capacity factor during the peak 50 hours of demand minus wind production. In Section 3.5 we find a better estimate for the capacity value of solar in each scenario using the results of our simulation.

We ran eight additional scenarios, referred to as dispersion tests, to more specifically study the effect of spacing between utility-scale PV systems and climate conditions. These scenarios consist of 100, $120 \mathrm{MW}$ PV systems randomly located in areas of different sizes: $50 \mathrm{~km}, 100 \mathrm{~km}$, $200 \mathrm{~km}$, and $300 \mathrm{~km}$ squares. We created two scenarios for each of these area sizes, one in the "best" available climate and one in the "worst". All areas used for the dispersion test scenarios are shown on the rightmost maps in Figure 1. We define the favorability of climate using a weighted average of three metrics: (1) frequency of sub-hourly cloud volatility, (2) frequency of hourly cloud volatility, and (3) expected annual capacity factor. These scenarios are meant to be illustrative but not realistic, and in order to achieve adequate dispersion we removed the ecological constraints. Section A of the supporting information further explains these placements.

The rooftop scenario distributes 2.4 million 5 kilowatt PV systems throughout California based on the density of residential structures within each census tract. ${ }^{4}$ Figure 2 displays lo-

\footnotetext{
${ }^{1}$ We define rated capacity by the AC power produced under $1000 \mathrm{~W} / \mathrm{m}^{2}$ of solar irradiance on the panel surface, after the balance of systems and other efficiency losses.

${ }^{2}$ For all non-rooftop scenarios, we assume that utility-scale PV systems have ground cover ratio of 0.15 and are aligned to face due south with East-West single axis tracking.

${ }^{3} \mathrm{Wu}$ et al. identify multiple different criteria for exclusion. At the recommendation of the authors, we use the second most stringent criteria - they identified the most stringent criteria as infeasible. These exclude all land already excluded by law, land at high risk for biodiversity loss, and land that contains unique natural characteristics, historical value, or cultural value.

${ }^{4}$ The census does not directly report the number of structures in each census tract, instead this value is estimated using the number of housing units per tract and the percentage of units in multi-unit buildings. Please see Section A of the Supporting Information for more detailed information.
} 
Table 1: Locational scenarios for $12 \mathrm{GW}$ of rated PV capacity in California.

\begin{tabular}{|c|c|c|c|}
\hline Scenario & $\begin{array}{l}\text { \# of } \\
\text { plants }\end{array}$ & $\begin{array}{c}\text { Plant } \\
\text { cap. }\end{array}$ & Location \\
\hline \multicolumn{4}{|c|}{ Utility-scale scenarios } \\
\hline 100-sys & 100 & $120 \mathrm{MW}$ & \\
\hline 50 -sys & 50 & $240 \mathrm{MW}$ & \\
\hline 25 -sys & 25 & $480 \mathrm{MW}$ & \\
\hline 4 -sys & 4 & $3 \mathrm{GW}$ & \\
\hline 1-sys (E) & 1 & $12 \mathrm{GW}$ & (E) energy value \\
\hline 1-sys (CV) & 1 & $12 \mathrm{GW}$ & (CV) capacity value \\
\hline
\end{tabular}

Dispersion tests, best climate

$50 \mathrm{~km}$

$100 \mathrm{~km}$

$200 \mathrm{~km}$

$100120 \mathrm{MW} \quad$ Best climate

$300 \mathrm{~km}$

Dispersion tests, worst climate

$50 \mathrm{~km}$

$100 \mathrm{~km}$

$200 \mathrm{~km}$

$100120 \mathrm{MW} \quad$ Worst climate

$300 \mathrm{~km}$

Rooftop $\quad 2.4 \mathrm{M} \quad 5 \mathrm{~kW} \quad$ Urban areas

No Solar

1,12 GW System

4, $3 \mathrm{GW}$ System

25, 480 MW Systems

100, $120 \mathrm{MW}$ Systems

Dispersion Tests

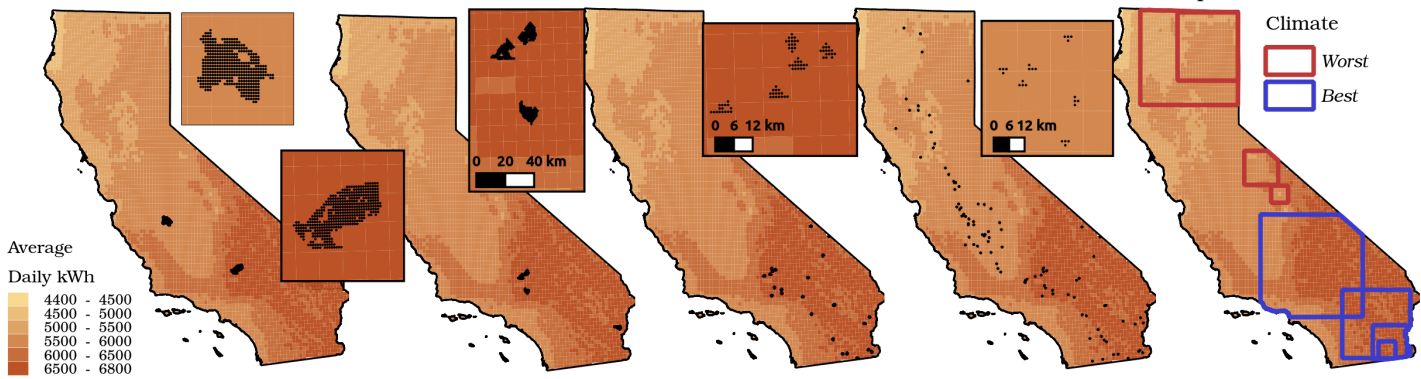

Figure 1: Utility-scale PV scenarios. Black dots represent $1 \mathrm{~km}$ grid cells, each of which contain $30 \mathrm{MW}$ of PV. The left panel contains scenarios where all $12 \mathrm{GW}$ are sited in one contiguous system, (E) located for the greatest energy production and (CV) located for the greatest capacity value. Locations are increasingly dispersed in each subsequent scenario, ending with 100,120 MW. The rightmost panel contains regions used for the dispersion test scenarios. 


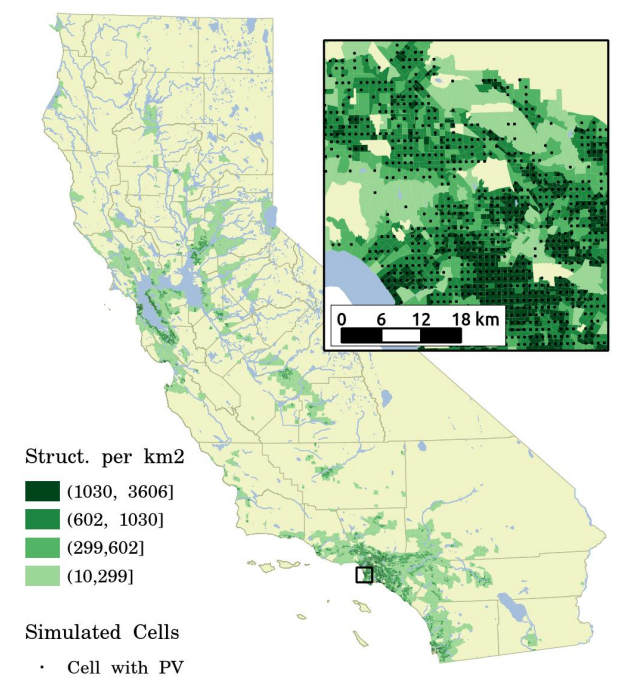

Figure 2: Map of the rooftop scenario of PV locations. Shades of green indicate the density of residential structures within census tracts, and black dots indicate $1 \mathrm{~km}^{2}$ grid cells in which PV systems are located.

cations for these rooftop systems on a coarse scale. Section A of the supporting information includes a more detailed account of PV placement in the rooftop scenario.

For the utility-scale scenarios, we also investigated two balancing policies: (1) The system operator balances the net load without differentiating between solar, wind, and load, and (2) The system operator balances wind and load, but solar plants balance themselves by independently procuring load following and regulation. The later scenario reflects policies where solar PV plants "smooth" their own generation using co-located controllable resources.

\subsection{Defining system balancing}

Wind and solar generators have nearly zero marginal cost and can be viewed as a load modifier, meaning that system operators will dispatch controllable resources to balance net-load $=$ load - solar - wind. ${ }^{5}$ Controllable resources include generators, energy storage and communicating demand-side resources. Many controllable resources have long start-up times or cannot change output quickly, e.g., large coal, nuclear, and to some extent combined cycle gas generators. To accommodate these resources the system operator schedules them to change their output at most once an hour. System operators then hold in reserve enough flexible capacity to respond, with high reliability, to the variability and uncertainty of net-load around this schedule. Deviations between supply and demand manifest as variation in power system frequency. The fastest reserve capacity will respond to these changes in frequency and are thus referred to as frequency regulation reserves. To limit regulating capacity requirements, system operators

\footnotetext{
${ }^{5}$ System operators may curtail renewable generators when controllable generators reach minimum output levels or net load becomes negative. Although these circumstances are rare today, they could become more prevalent as more renewable generators are connected. As we will explain, this curtailment can be seen a flexibility product itself that is separate from net-load.
} 


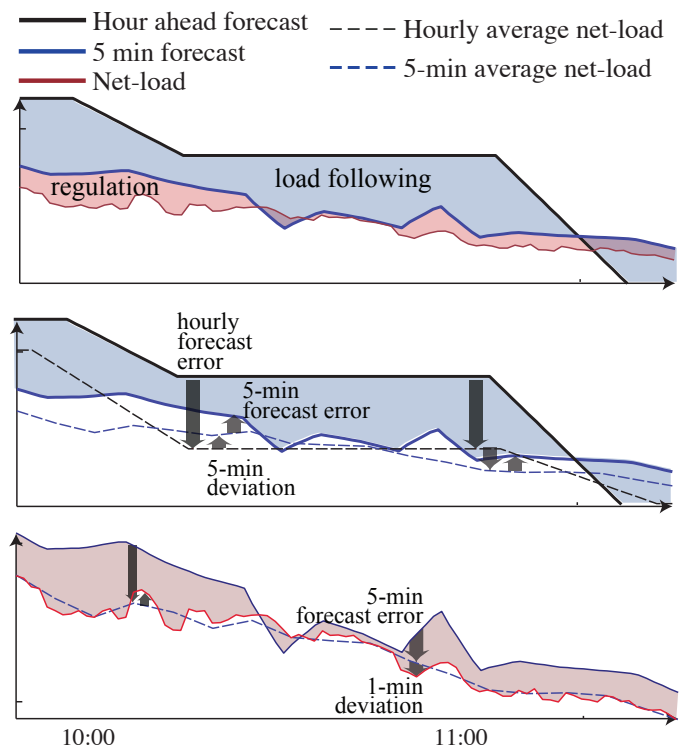

Figure 3: Quickly-responding controllable resources, set aside as reserves, balance net-load as it deviates from hourly scheduled generation. (Top) load following reserves follow a 5-min schedule as it deviates from the hourly schedule, and regulation reserves respond to grid frequency to balance real-time load as it deviates from the 5-min schedule. (Middle) load following reserves effectively compensate for hourly forecast errors, 5-min forecast errors, and 5-min deviations. (Bottom) regulation reserves compensate for 5-min forecast errors and 1-min deviations.

may run an intermediate market that includes resources that do not provide frequency regulation but are available to respond faster than the hourly schedule. This intermediate responding capacity is often referred to as load following reserves. Figure 3 shows both load following and regulation reserves as the difference between schedules: load following is the difference between hourly and 5-min schedules, and, regulation is the difference between actual net-load and the 5 -min schedule.

Reserves compensate for uncertainty and variability of net-load. Uncertainty is the inability to perfectly predict net-load, resulting in erroneous schedules. Variability is the result of each subsequent schedule changing more quickly than its predecessor, even when both are based on perfect forecasts. Please see Makarov 2009 for a more complete explanation [29]. Our analysis rests on simulating four metrics of uncertainty or variability of solar, wind, and load:

Hourly forecast errors, $\epsilon_{60,60, t}$ : errors in a forecast of hourly average demand or generation.

5 minute deviations, $\eta_{5,60, t}$ : the differences between a 5 minute and an hourly schedule that are based on perfect forecasts. This is constructed to contain only variability and not uncertainty.

5 minute forecast errors, $\epsilon_{5,5, t}$ : Errors in a 5 minute forecast based on persistence of a the 
clearness index.

1 minute deviations, $\eta_{1,5, t}$, deviation of 1-min generation/demand from a 5-min schedule based on a perfect forecast. ${ }^{6}$

The middle and bottom panels of Figure 3 show how reserves are composed of uncertainty and variability. Section B of the supporting information shows mathematically what we show graphically in Figure 3: the use of load following reserves is the sum of $\epsilon_{60,60}(t), \eta_{5,60}(t)$ and $\epsilon_{5,5}(t)$, and the use of regulation reserves is the sum of $\epsilon_{5,5}(t)$ and $\eta_{1,5}(t)$.

It may seem counterintuitive that 5 minute forecast errors should affect both regulation and load following reserves. As a descriptive example of this double counting, consider what will happen if a 5-minute forecast predicts that demand will increase, but instead it remains constant: load following reserves will increase generation in order to meet the erroneous forecast, and regulation reserves will reduce output in real time to make up for the unnecessary increase in load following. System operators must account for these forecast errors when planning both the load following market and frequency regulation.

Our method is general and can be applied to any set of timescales. For this study, we chose the time-scales previously used by the California system operator to run energy and reserve markets, as defined in [29]. In response recent regulations, ${ }^{7}$ CAISO and many other utilities in the Western United States now reschedule generation on a 15 minute basis rather than hourly. Forecasts for these new markets are made 40 minutes in advance of the interval, thus forecasts are made 47.5 minutes before the midpoint of the interval. This makes them not so dissimilar to the hourly forecasts that we use, which are made 30 minutes in advance of the midpoint of the interval.

In contrast to our analysis, 15 minute schedules ramp continuously over each interval rather than plateauing in the middle; thus new schedules will better match predictable changes of netload over time, such as the effect of sunrise and sunset on solar generation. In Section 3.2.1, we remove the effect of predictable variations on reserves to better model the effect of this new scheduling.

\subsection{Simulating PV}

We modified our model from [23] to simulate variability and uncertainty of PV generation in each locational scenario. In this section we provide a brief explanation of the model framework, including updates. Appendix $\mathrm{C}$ of the supporting information contains a detailed description.

Figure 4 (left) shows a graphical depiction of dependencies in our model, using variables that we explain in this section. This structure is applied to generate models for each of the four metrics variability or uncertainty defined in Section 2.2. To explain the structure, we generally refer to these metrics as $y_{i, t}$; subscripts signify the metric from PV system $i$ at time $t$.

Our primary innovation is that we condition the standard deviation of $y_{i, t}$ on an endogenously estimated latent state, $v_{i, t}$, referred to as a volatility state - defined and fully explained in Section C.1. The volatility state for each PV system allows for rapid transitions between periods of high standard deviation and low standard deviation. Figure 4 (center) shows how

\footnotetext{
${ }^{6}$ Note that our data do not support investigations of deviations on time scales faster than one minute, but if such data were available this metric could constructed on the relevant time scale - e.g. if 4 second data were available one would compute 4 second deviations from the 5 minute schedule.

${ }^{7}$ Order 746 of the Federal Energy Regulatory Commission.
} 


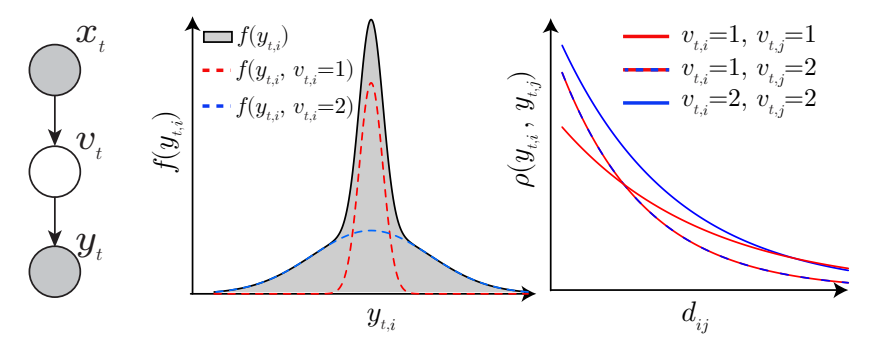

Figure 4: Left, Graphical model representing dependence between variables. Center, a pdf of variability or uncertainty at one PV system, $y_{t, i}$, represented as a mixture of Gaussians. Volatility states, $v_{i, t}$, define which mixture component is exhibited. Right. Correlation of $y$ between two systems, $\rho\left(y_{t, i}, y_{t, j}\right)$. Correlation decays exponentially with distance, the parameters of this exponential decay are dependent on the volatility states of both systems.

Table 2: Summary of parameters for modeling variability and uncertainty from PV.

\begin{tabular}{|c|c|c|c|c|}
\hline \multirow{2}{*}{$\begin{array}{c}\text { Variability / } \\
\text { Uncertainty metric }\end{array}$} & \multicolumn{2}{|c|}{ (\% of clear sky) } & \multicolumn{2}{|c|}{ rate $(\mathrm{km})$ weighted } \\
\hline & range & mean & range & mean \\
\hline Hourly forecast errors, $\epsilon_{60,60}$ & {$[6.3,21.9]$} & 11.8 & {$[46.2,62.5]$} & 53.34 \\
\hline 5 -min forecast errors, $\epsilon_{5,5}$ & {$[0.08,22.1]$} & 1.6 & {$[0.10,60.7]$} & 2.48 \\
\hline 5-min deviations, $\eta_{5,60}$ & {$[0.37,16.4]$} & 3.2 & {$[0.10,20.2]$} & 9.6 \\
\hline 1-min deviations, $\eta_{1,5}$ & {$[0.18,16.1]$} & 2.0 & {$[0.10,2.21]$} & 0.87 \\
\hline
\end{tabular}

the distribution of $y_{i, t}$ is a mixture of Gaussian distributions. The mean of $y_{i, t}$ is 0 for forecast errors, signifying that forecasts are unbiased; for deviations the mean is defined as the deviations of a deterministic clear sky signal.

$y_{i, t}$ from neighboring systems are spatially correlated, with correlation coefficients dependent on volatility states of both systems. Figure 4 (right) shows multiple spatial correlation functions. One function is estimated for each possible pairing of volatility states.

The distribution of $v_{i, t}$ depends on a set of climate variables, $x_{t}$, which can be any set of discrete observations from the geographic areas and time periods modeled. In this study, $x_{t}$ represent cloud variability in $1 \mathrm{~km}^{2}$ grid cells, and are derived from measurements of global horizontal irradiance provided to us by Clean Power Research. ${ }^{8}$

Model parameters are fitted using observations of both $y_{t}$ and $x_{t}$; values of $v_{t}$ are latent, meaning that they are never observed but are estimated during the fitting process. Simulation requires only observations of $x_{t}$, the locations of PV systems, and the parameters are required. Distributions of $y_{t}$ and $v_{t}$ are produced by the simulation; explained in Section C.

Table 2 presents ranges and time weighted averages for standard deviations and correlation decay rates for each metric. Noticeably, hourly forecast errors exhibit relatively large standard deviations and long correlation decay rates, indicating that they will have the greatest effect on reserve needs.

Utlity-scale PV plants in our scenarios range from $120 \mathrm{MW}$ to $12 \mathrm{GW}$. However we fit our models using systems that only range from $2 \mathrm{~kW}$ to $12 \mathrm{~kW}$. We expect that there will

${ }^{8}$ Full derivations of the climate data are included in Sections D.4 and D.3 of the supporting information. 


\footnotetext{
${ }^{9}$ This arrangement results in $30 \mathrm{MW}$ of $\mathrm{PV}$ capacity per $\mathrm{km}^{2}$, which is similar to land use efficiencies found in a recent survey of utility-scale solar installations [30].
}

be significant effects of geographic diversity within the utility-scale systems beyond what we observed in the distributed systems. We account for this intra-plant geographic diversity by modeling utility-scale PV systems as collections of $5 \mathrm{~kW}$ systems arranged in grids that are spaced such that the ground cover ratio is 0.15 at a panel efficiency of $0.20 .^{9}$ In all cases, the utility-scale systems also span more than $1 \mathrm{~km}^{2}$, and thus are affected by multiple observations of climate at each time. Thus our simulations account for geographic smoothing and varying climate within each utility-scale plant.

Reducing the computational costs of the simulation was necessary for this study. Each of our locational scenarios requires simulating thousands of $M \times M$ covariance matrices, where $M=2.4$ million $5 \mathrm{~kW}$ PV systems. This simulation is theoretically possible however is computationally infeasible on common computers. To reduce computational costs, we make two approximations, which allow us to run simulations in two, tractable stages. Our approximations and our two-stage method are fully explained in Section $\mathrm{C}$ of the supporting information.

Predicting hourly forecast errors with our model is a new innovation in this paper that we fully explain in Section D.2 of the supporting information. In brief, hourly forecasts are generated using a linear model conditioned on lagged values of solar generation, the expected solar irradiance given a clear sky, and time of day and seasonal fixed effects. From our model, we simulate a root mean square error (RMSE) of between 9.6 and 11.4 MW for the $120 \mathrm{MW}$ plants in our scenario with 100 utility-scale systems. This is consistent with or better than commercial forecasts evaluated in [31]. The success of our simple regression model is unsurprising for hourahead predictions, for which persistence forecasts are difficult to out-perform [32]. In order to be consistent with our approach to the sub-hourly metrics, we used our volatility state model to predict the hourly forecast errors for each locational scenario. However, in exploratory work, we found that the volatility state model performs as well as one without latent states for these hourly metrics. We describe this result in Section D.7 of the supporting information.

The contribution of PV to reserves is calculated by adding forecast errors to deviations, again resulting in Gaussian mixture distributions. We assume no correlation between forecast errors and deviations given the volatility states, however we do assume correlation of the volatility states. Thus forecast errors and deviations are more likely to be volatile at the same time than if they were independent, but are not more likely to fluctuate in the same direction. Section C.4 of the supporting information explains this method for summing the forecast errors and deviations, and validates our simulation method using data from model fitting. Overall, our method accurately predicts the distributions of load following reserve use, and it provides a conservative estimate of the distribution tails of regulation reserve use.

\subsection{Variability and Uncertainty from Wind and Load}

The CAISO provides one-minute resolution wind generation and load data for the year 2005 . We obtained these data and then linearly scaled them to represent growth between 2005 and 2020, when California's independently owned utilities are required to procure $33 \%$ of energy from renewable sources. For load, we used an annualized growth rate of $1.5 \%$, resulting in a scaling factor of 1.25 over 15 years. For wind, we scaled it to match current projections for wind energy in California as presented in a recent long term planning paper, [27]. These scaled data are used 
to create one year of variability metrics $-\eta_{5,60}$, and $\eta_{1,5}$ - for wind and load in the year 2020 . We simulate forecast errors $-\epsilon_{60,60}$, and $\epsilon_{5,5}$ - for both wind and load using an autoregressive processes defined in [29] and used in CAISO's recent renewables projections. ${ }^{10}$

We adapt a sampling method put forward by $\mathrm{Wu}$ et al. in [1] to simulate reserve needs from wind and load during our study period. We bin all wind generation data by the decile of hourly average wind generation, implying that wind may be more or less variable depending on the average generation. We bin load data using only hour of day and month of year, capturing diurnal and seasonal changes in load variability. For each bin, we create a mean profile within the hour; this effectively captures steady increases or decreases in load during the morning or evening. We then store all observed deviations from this mean profile. A sample of wind or load's contributions for reserves then includes the mean profile plus randomly sampled deviations. I.e. the load following reserve need at 10:35 am on Jan 2 is simulated as the average of all needs at 10:35 in January, plus a random selection of any of the deviations observed between 10:00 and 10:55 in January.

We include variability and uncertainty from wind and load to mimic the context of a larger system, but these are not the focus of our study and thus we are less detailed in their modeling. We list here some limitations of our wind and load models. (A) Our model does not account for geographic diversity of wind generators. Thus we expect our linear scaling of wind generation to over-estimate variability. In the results section we include two sensitivity analyses to test how reducing the variability from wind and load affects our findings. (B) Our method also does not account for changes in the timing of consumer demand schedules. (C) Finally, coincident patterns in the solar, wind and load data are reflected in our analysis only insofar as they are contained in the input data. Beyond this, variability and uncertainty from solar, wind, and load are simulated independently.

\subsection{Procuring reserves}

Figure 5 shows the process of simulating reserve procurement from the solar, wind, and load simulations. We use the models described above to create probability distribution shapes for wind, load, and PV's contributions to reserves. From these distributions, we sample 200 realizations at each appropriate interval: 1-min for regulation and 5-min for load following. We calculate the reserves needed to follow net-load by subtracting solar and wind's contributions from load's contribution. This creates 200 equally likely values for reserve need at each interval in our study year.

We procure reserves such that they are sufficient in $95 \%$ of hours. This is achieved by defining reserve "up" and "down" procurement as the $97.5^{\text {th }}$ and $2.5^{\text {th }}$ percentiles of reserve needs realized in that hour.

We condition the distributions of wind and solar reserves on information that will only be available during the period reserves are used, and not during the earlier time at which reserves are procured. A system operator must rely on less certain forecasts, and will be less able to determine when more or less reserves are needed. As a result, we expect our estimates for hourly reserve procurement to be more variable than those of an actual system operator. However, conditioning variables are coarse and potentially easy to predict: there are only 10 possible deciles of hourly average wind generation, and only 5 possible climate states for solar. Thus we

\footnotetext{
${ }^{10} \mathrm{We}$ describe this method in more detail in [33].
} 


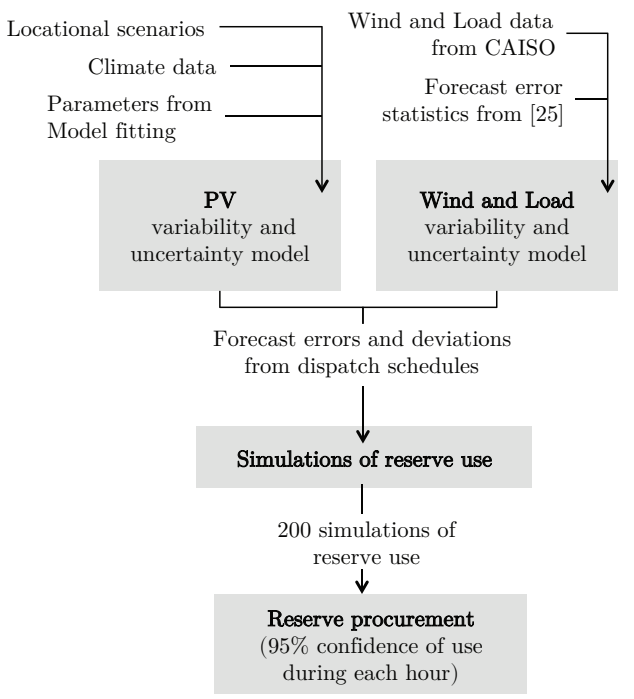

Figure 5: Process diagram for the construction of load following and regulation reserve signals

do not expect this assumption to greatly affect our results.

\section{Results}

\section{$3.1 \quad$ Net-load}

Figure 6 shows four example days of net-load and renewable generation from the utility-scale and rooftop scenarios. The rooftop scenario generally produces less overall generation than the centralized scenarios, because rooftop systems don't track the sun, have less ideal geometries, and are not sited in areas with the greatest energy resource.

Notably, morning and afternoon ramps in PV generation - those ramps resulting from sunrise and sunset - have opposite effects on generation ramping requirements. On some days, the morning rise in PV generation mitigates a simultaneous rise in demand reducing the overall ramp in net load. The afternoon drop in PV generation exacerbates the power ramp already needed to meet an afternoon peak in demand - this is most pronounced in colder months (January and April). This effect is well known to system planners, and commonly referred to as the duck curve.

\subsection{Hourly reserve procurement}

Figure 7 shows the minimum, mean, and maximum of hourly reserve procurement for each scenario. We compare scenarios by how much reserve procurement increases from the baseline scenario without solar. Hourly reserve procurements depend strongly on spatial configuration: 


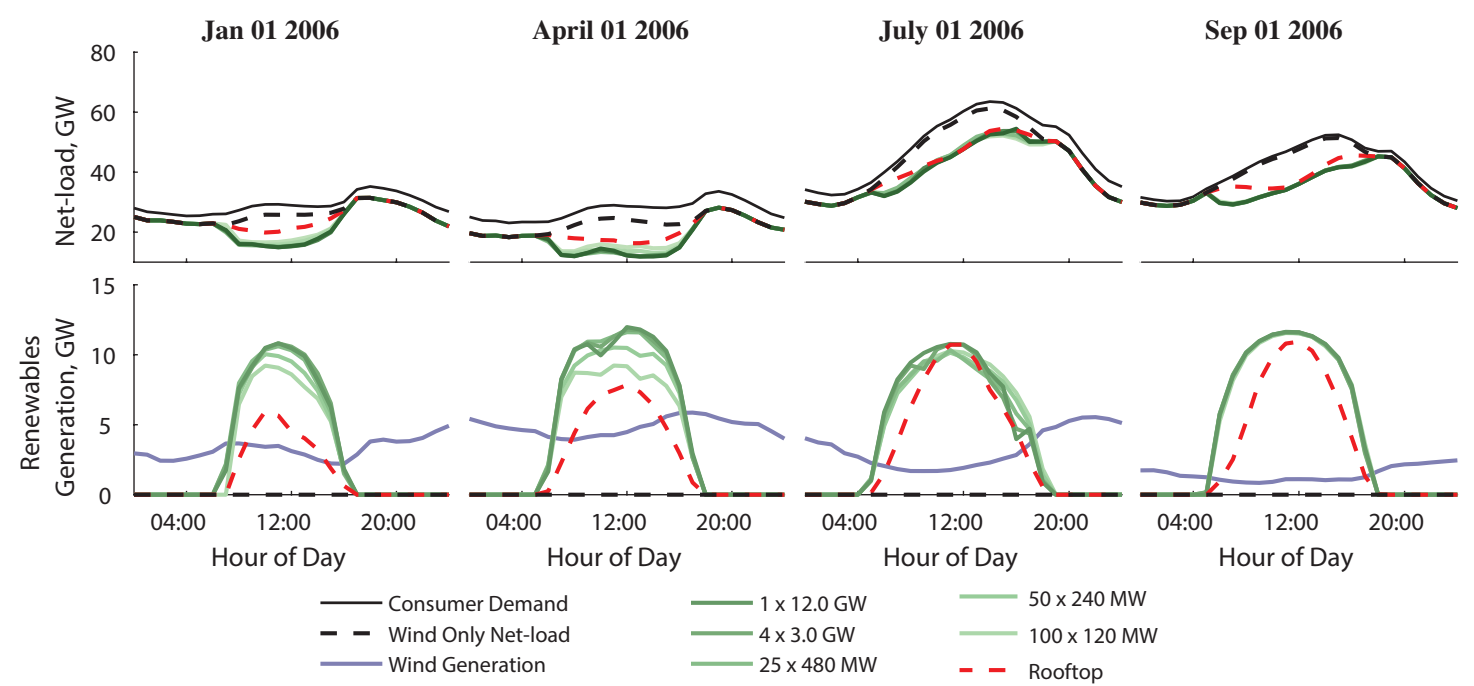

Figure 6: Net-load and renewable generation for four days of the utility-scale, rooftop, and baseline scenarios.

the load following reserve increase in the worst utility-scale scenario (single $12 \mathrm{GW}$ system sited to maximize capacity value) is 4 times larger than in the best utility-scale scenario. Though the maximum percentage increase in frequency regulation reserve is smaller than for load following, the range of percentage increases is more pronounced because the strongly dispersed cases result in essentially no increase in reserve requirements.

On average, reserve requirements are greater when we site a single $12 \mathrm{GW}$ system to maximize capacity value rather than energy production (see the "CV" case versus the "E" case). This is because the CV site has a higher total fraction of partly cloudy hours. We will show later, however, that at peak hours the need for reserves at the CV site is lower than at the E site.

As one might expect, the dispersion tests show that the size of the area over which systems are distributed clearly matters. By constraining the 100 systems to small areas (50 or $100 \mathrm{~km}$ squres), almost all of the benefits of dispersion are negated. However reserve reductions diminish as the size increases. Constraining systems to be in areas with different climates has large effect on concentrated arrangements, but a negligible effect on dispersed arrangements.

The right-most scenarios in Figure 7 show that requiring individual plants to balance their own output locally - for example with energy storage - significantly increases reserve capacity requirements. Balancing locally removes two benefits of aggregation. First, solar is balanced separately from wind and load, and this is the driver for the increase in reserve requirements in the single system cases. Second, the benefits of decaying spatial correlation are lost, which explains why the reserve requirement increase is greatest for the 100 system case. Taken together these results indicate that that local balancing is likely to be very expensive if balancing costs scale linearly with required capacity.

\subsubsection{Sensitivity analyses}

Figure 8 shows the results from four sensitivity cases that we ran to better qualify our results. Black dots in Figure 8 show our original estimates of load following reserve need for the baseline and utility-scale scenarios; these match the values presented in Figure 7. 

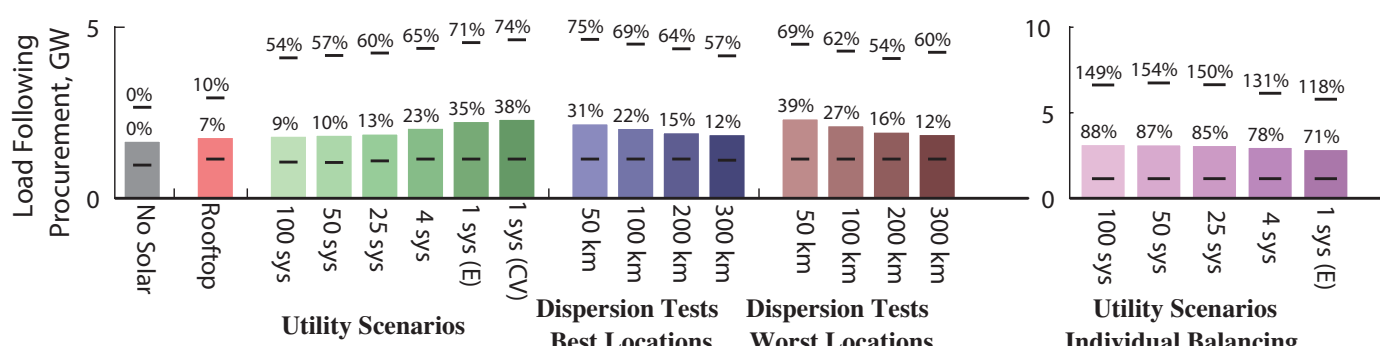

Utility Scenarios Individual Balancing
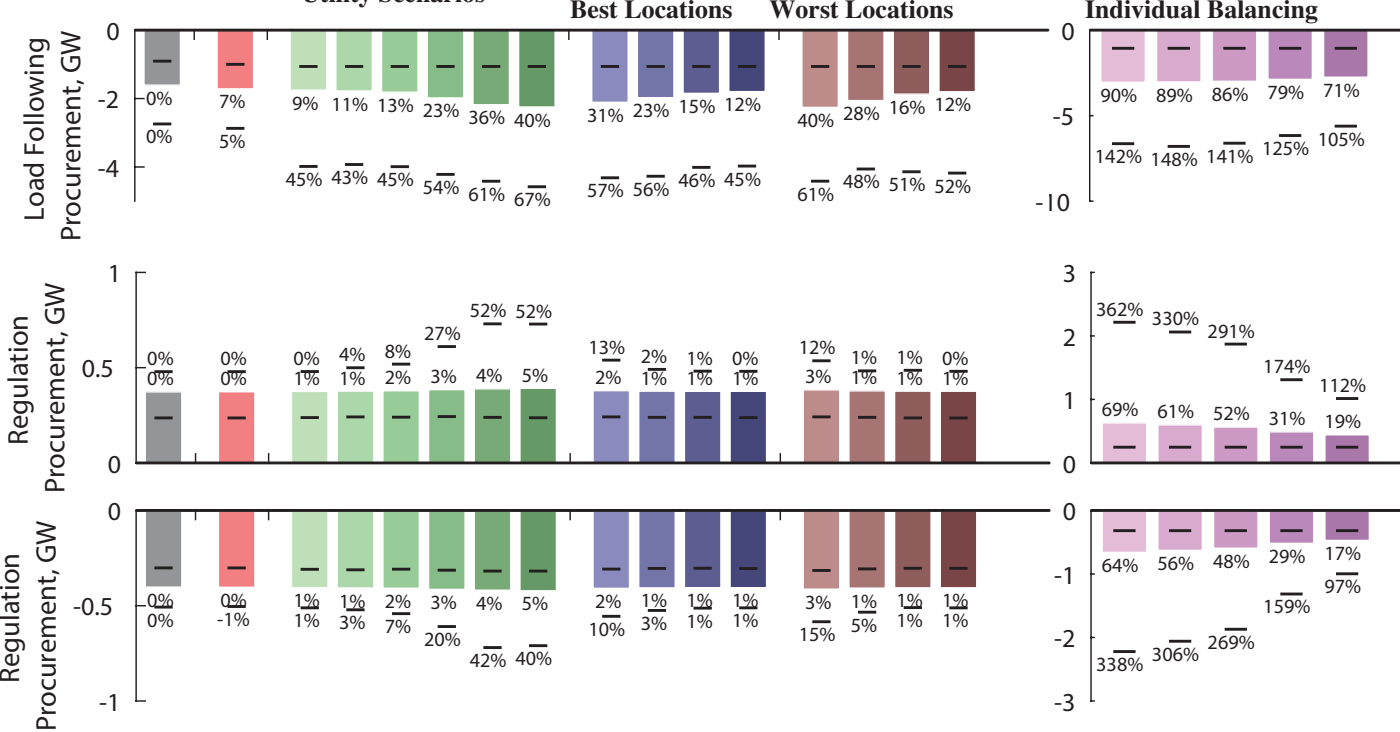

Figure 7: Average hourly reserve procurement in each scenario. Lines above and below each bar represent the maximum and minimum reserve procurement. Labels above each bar are the percent change in average procurement from the baseline scenario; labels above each maximum line are the percent change in the maximum procurement of reserves. 


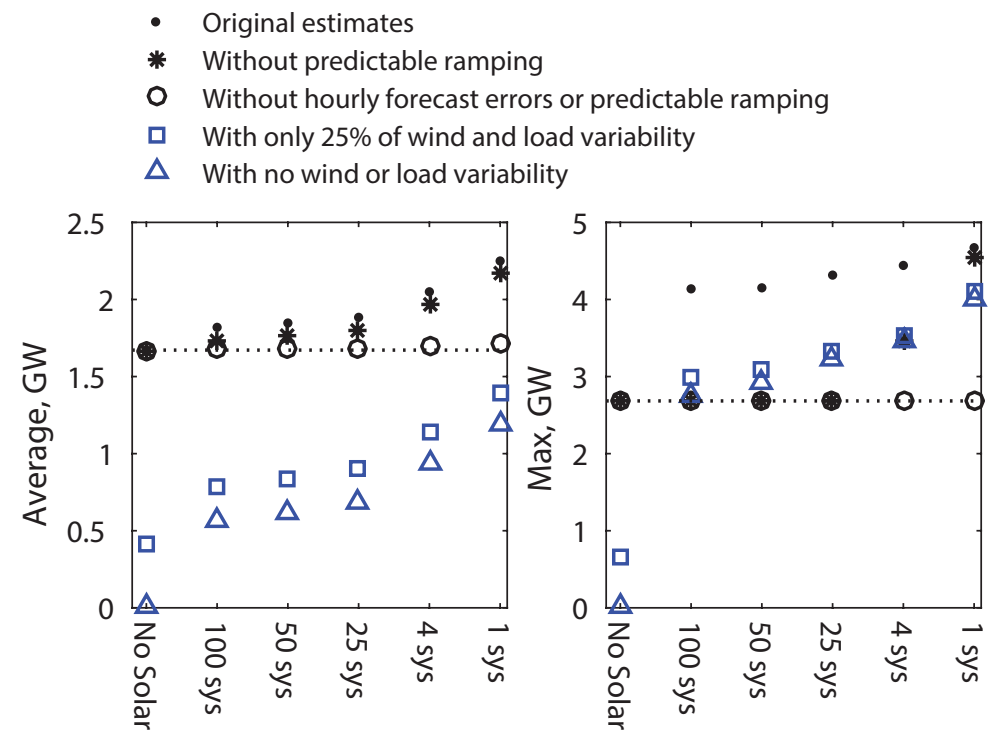

Figure 8: Average and maximum hourly load following procurement for the baseline and utility-scale scenarios, original results are presented along with four sensitivity cases.

One major driver for load following reserves is predictable changes in net load throughout each hour. The CAISO's updated 15 minutes schedules ramp continuously rather to plateau within each hour, and are better able to match these changes without the need for load following reserves. To mimic 15 minute schedules, we remove the average load following need from solar. In order to maintain a consistent baseline from which we assess the effects of solar variability we do not remove the mean from wind and load.

Black stars in Figure 8 present results from scenarios when the mean is removed. Average procurement reduced by about $80 \mathrm{MW}$ in all scenarios, $\approx 5 \%$ of baseline. Maximum procurement reduced to that in the baseline case for all except the two most centralized scenarios, ${ }^{11}$ signifying that the large increases in maximum procurement were driven entirely by the steep morning and evening ramps of utility-scale solar.

Black circles in Figure 8 display results from our analysis when forecast errors are also set to zero. We find that forecast errors account for between $90 \%$ and $95 \%$ of the reserve increases from solar in all scenarios - once the predictable solar diurnal cycle is also removed.

Figure 8 also contains two scenarios that test the effect of variability from wind and load: one where we include only $25 \%$ of this variability (blue squares) and one where we include none of it (blue triangles). We find that the inclusion of solar in general has a greater relative impact in these cases, as seen by comparing the baseline case to all other cases. However our general findings regarding spatial diversity remain: there are diminishing returns to dispersion,

${ }^{11}$ This maximum occurs at $6 \mathrm{pm}$ in the winter, during the evening peak in consumption depicted in Figure 6 


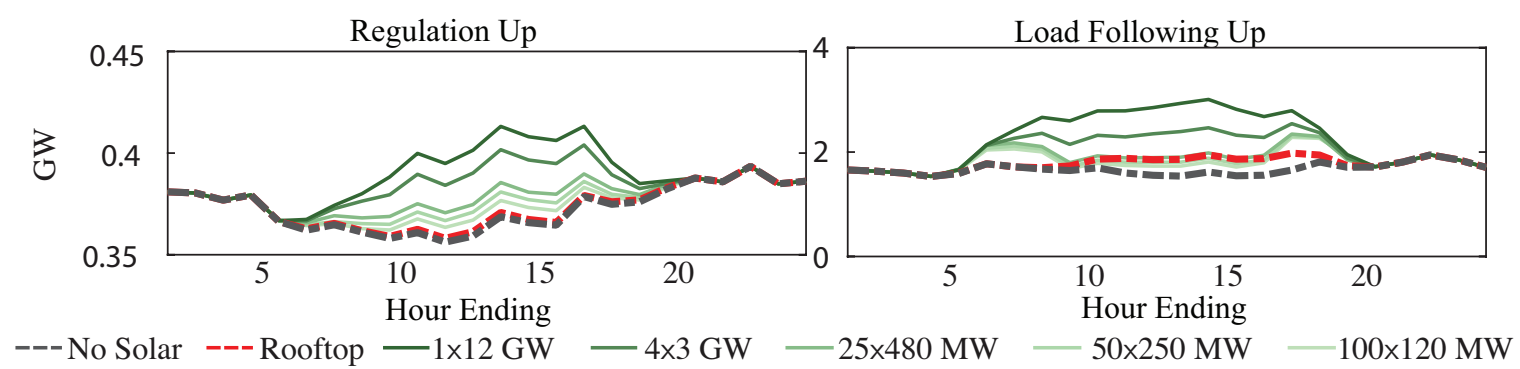

Figure 9: Average regulation and load following reserves procured in each hour of day for the utility-scale, rooftop, and baseline scenarios.

particularly after the 25 system scenario.

\subsection{Diurnal variations in reserve need}

The solar diurnal cycle drives not only the generation but also magnitude of variability and uncertainty in PV. Averaging the effect of PV on reserve need throughout the year belies the consistently larger effect PV will have at midday when potential generation is greatest.

Figure 9 shows the average procurement of load following and regulation reserves stratified by hour of day. As expected, reserve increases in the solar scenarios are greatest at midday, although these increases are rapidly mitigated by geographic diversity. There are consistent increases in load following reserve requirements in the morning and afternoon that are not mitigated by geographic diversity. This is due to the predictable ramping of the solar diurnal cycle, and will be mitigates with more flexible generation schedules.

\subsection{Curtailing PV to provide reserves}

Renewable generators may provide reserves by dynamically curtailing generation, as suggested in $[34,35]$ and currently practiced by the Public Service Company of Colorado [36]. In this section we estimate the amount of PV energy that must be curtailed to provide the reserve procurement that exceeds that in the baseline scenario. While there will often be less expensive reserve capacity than curtailing solar, this estimate provides a conservative boundary for the costs of variability and uncertainty that PV adds to supply.

To provide up reserves, a PV system must pre-curtail power output by the amount of up reserves contracted and reduce this curtailment to reliably increase generation when needed. To provide down reserves, a PV system could simply curtail power output to reduce its own generation when needed. As in [35], we conservatively assume that $25 \%$ of the power committed to up reserves is used on average. ${ }^{12}$ Thus, PV will curtail only $75 \%$ of the MW-h committed to up reserves in a given hour. Analogously, we assume that $25 \%$ of down reserves committed will be

\footnotetext{
${ }^{12}$ Assuming that up and down reserves are mutually exclusive and equally likely, each have a $50 \%$ likelihood of being called. Given that a set of reserves are called, we conservatively assume that $50 \%$ of committed capacity is used on average. The result is $.5 \times .5=25 \%$ of committed capacity being used on average.
} 


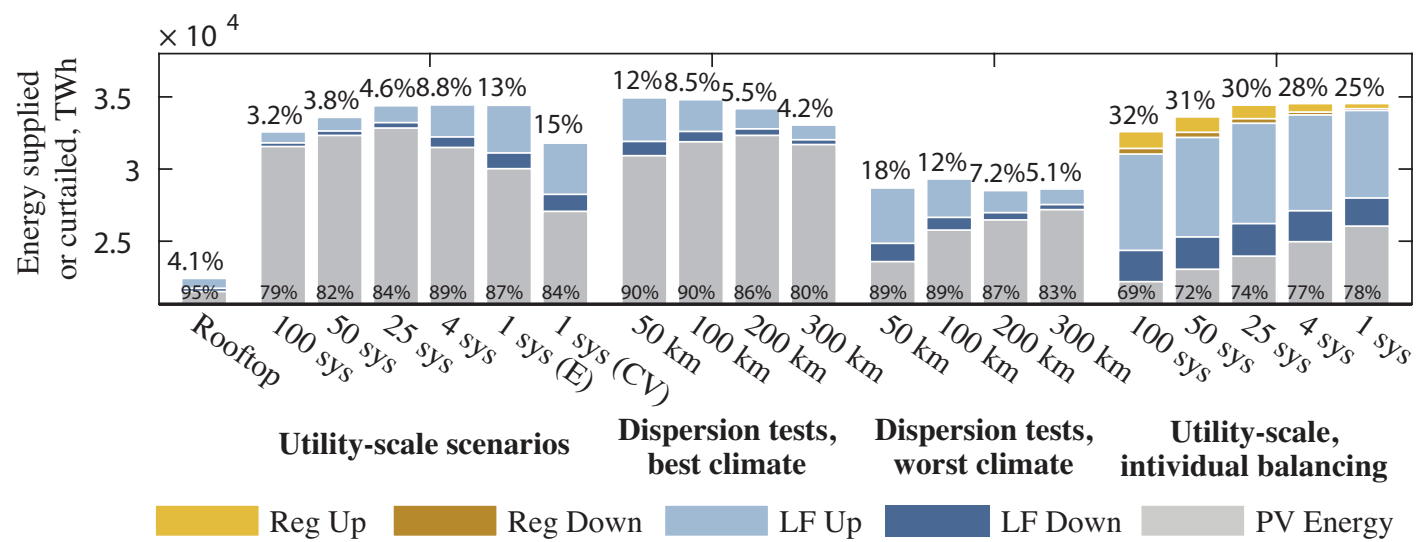

Figure 10: Total energy produced in each scenario, and energy curtailed to provide additional reserves resulting from the inclusion of solar. The percentage of PV energy curtailed in order to provide reserves is labeled above each bar; The percentage of additional reserves able to be supplied by curtailing PV is labeled below each bar.

curtailed on average. ${ }^{13} \mathrm{PV}$ systems can only commit as much capacity as they reasonably expect to produce consistently throughout an hour. We do not allow PV to commit more than $70 \%$ of its hourly average production to reserves; as a result, PV is insufficient to provide additional reserves in some hours.

In Figure 10 the height of each stacked bar shows the total PV energy that could be supplied annually in each scenario. Colored bars at the top of each stack represent PV energy that must be curtailed to provide each type of reserves. Thus, the bottom gray bar represents PV energy supplied after curtailment. Percentages above each stack signify the percent of PV energy curtailed, while percentages below each stack are the amount of hours that enough PV energy is available to provide the additional reserves (excluding hours with no PV generation). Typically, $\mathrm{PV}$ is insufficient to meet additional reserves in the early morning or afternoon hours, when both PV generation and the additional reserve need are low. ${ }^{14}$

More concentrated arrangements are able to supply more energy, but they also increase the need for reserves and thus increase curtailment. This trade-off is most apparent for the dispersion tests in the best climates: though potential PV generation is greatest when concentrating systems in a $50 \mathrm{~km}$ square area, $12 \%$ of this energy is curtailed to provide reserves. Conversely, in the most dispersed $300 \mathrm{~km}$ scenario there is less total potential for PV generation, but also less is curtailed for reserves. The $200 \mathrm{~km}$ scenario provides the optimal net contribution of energy among the dispersion tests in the best climates.

\footnotetext{
${ }^{13}$ If up and down reserve procurement are symmetric, then total curtailment will always be equal to total amount of reserves procured in one direction. This is true regardless of the percentage of committed reserves employed on average. I.e., if $x \%$ of committed up and down reserves are used on average, then up reserves curtail $(100-x) \%$ of committed power on average, and down reserves will curtail $\mathrm{x} \%$; thus the total reserve procurement adds to $100 \%$ of up or down procurement.

${ }^{14}$ During night-time hours PV doesn't generate energy but, as a result, doesn't contribute to reserves need. Thus there is no increase in reserve need during these hours.
} 


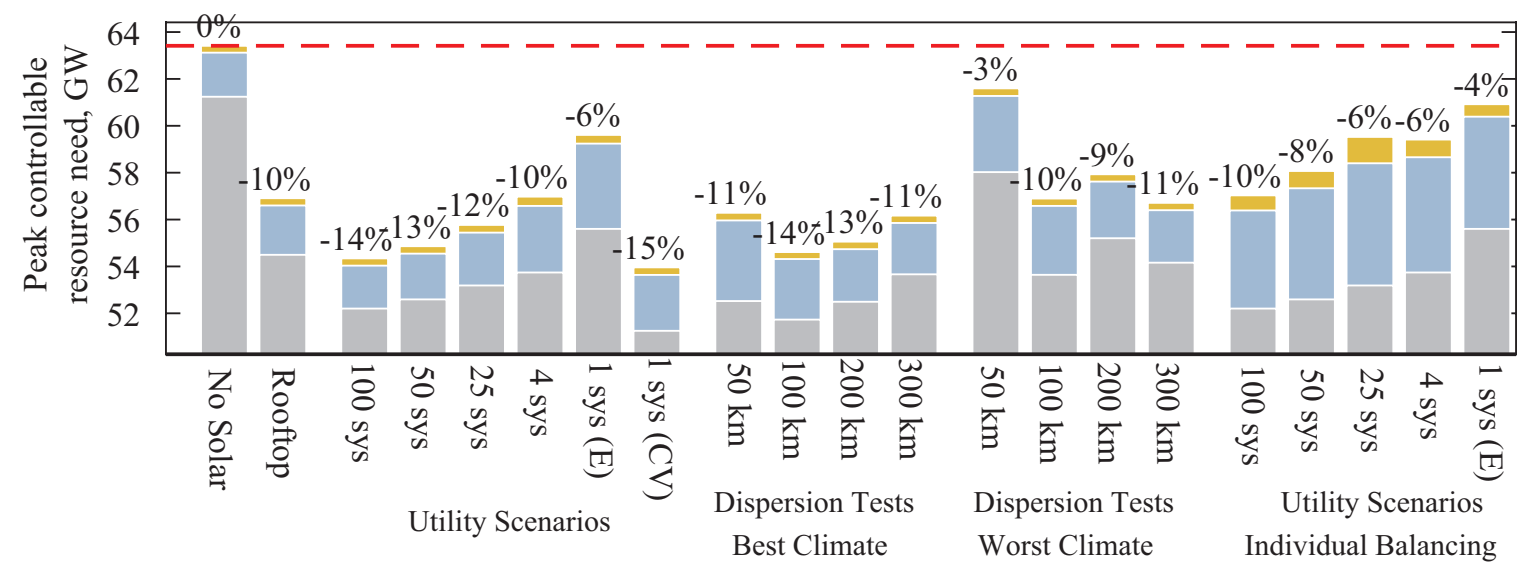

Reg Up

LF Up

Net-load

Figure 11: Maximum hourly need for dispatchable generation during the 2020 study year. Need for generation is broken by net-load (grey), load following up need (blue), and regulation up need (red).

\subsection{Capacity value of PV}

The capacity value of a resource reflects its ability to reduce the peak need for controllable resources - either generation, demand response, or storage - in the system. Solar generation may affect peak need for controllable resources in counteracting ways: first it may reduce net-load during peak hours, and second it may increases the need for reserves during peak hours.

We define the need for controllable resources as the sum of hourly net-load, load-following up procurement, and regulation up procurement; down reserves are excluded because controllable resources are conventionally generators, and additional generation capacity is not needed in order to provide down reserves. We define the capacity value of solar in each of our scenarios as the reduction in this peak as compared to the baseline scenario.

Figure 11 displays peak need for controllable resources in each scenario. Percentages above each bar represent the reduction from the baseline. The reduction in peak demand for controllable resources varies widely by scenario: from $3 \%$ to $15 \%$ of the baseline scenario, which is $15 \%$ to $80 \%$ of rated PV capacity.

Surprisingly, the scenarios in which PV is sited to achieve the greatest annual energy production have relatively small capacity values. This is most stark when comparing the 1-sys to 100-sys utility scale scenarios sited for greatest energy production, for which capacity value increases with dispersion. However, dispersion is certainly not necessary: by siting one system intentionally for its capacity value, it achieves the best results overall.

Solar resource data from the National Renewable Energy Laboratory (NREL) suggest that this is a general trend beyond our 2005 study year. The NREL data contain annual and monthly average PV resource, which is derived from satellite observations collected from 1998 to 2009. Figure 12 presents the annual averages along with averages for three summer months. On an annual basis, the Mojave Desert in the Southeast of the state contains a far greater solar resource. But in the months of July and August - which have most often contained peak demand [37] - the Central California valley and even some of the mountainous Northern California regions have a greater resource. Some areas in the Northern Mojave offer optimal mixes: large resources 


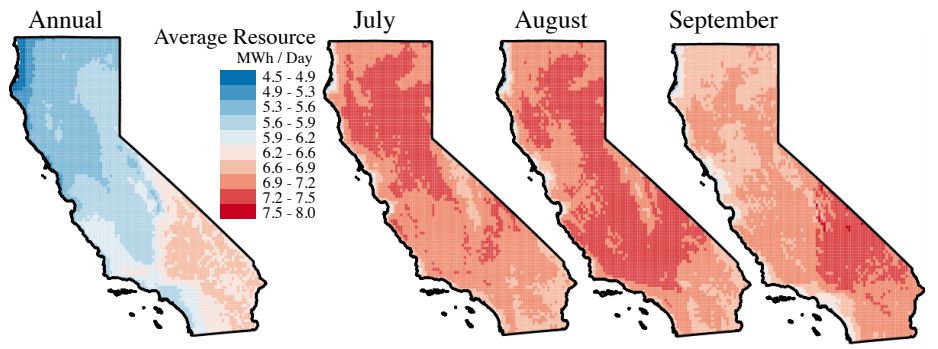

Figure 12: Average daily PV generation by region and month.

over the year and during summer months. Together, these data suggest a trade off between maximizing total energy, and energy during peak hours, where a few regions are optimal for both.

Note that our capacity value results are dependent on assumptions of how much renewable generation is included in the system and the timing of peak demand (we assume that load shapes are linearly scaled from 2005). California's future renewable energy goals are far more ambitious than their $33 \%$ goals modeled here, thus we expect there to be more wind and solar included eventually. The timing of peak demand will likely change in the future, particularly if end-uses change significantly, for example due to heating or transportation electrification.

\section{Discussion}

On avaerage, 100 dispersed PV systems require $500 \mathrm{MW}$ less reserves than 1 concentrated system. When dispersed, these $100 \mathrm{PV}$ systems increase load following reserve need by a small amount over a baseline case with no solar: $150 \mathrm{MW}$ on average, $9 \%$ of baseline. And they increase regulation reserves a negligible amount: $4 \mathrm{MW}$ on average, $1 \%$ of baseline.

As predicted in prior studies, the benefits of this dispersion can be achieved with relatively few systems $[10,38]$. We find that returns diminish beyond 25 plants, which in our study were almost $500 \mathrm{MW}$, the approximate size of the largest PV system in California today. However, systems must be adequately dispersed. Hourly forecast errors are spatially correlated even at distances of 40-60 km, and we find that constraining systems into small areas increases the need for reserves.

This study's results should be considered in policy discussions over where to construct PV or build additional transmission. Currently, more than $2 \mathrm{GW}$ of PV are already contracted within a $700 \mathrm{~km}^{2}$ area in the Tehachipi Competitive Renewable Energy Zone (CREZ) in California, and four other CREZs each contain more than $1 \mathrm{GW}$. Thus, when planning for the costs and benefits of integrating PV, systems sited in Tehachipi or other saturated areas will require additional costs for reserves than those in other CREZs ${ }^{15}$ [39]. Accounting for these costs requires updating the existing tools used for transmission planning, which do not model spatial correlation [8].

Hourly forecast errors are, by far, the greatest contributor to load following reserves. The second largest are predictable, diurnal ramps in PV generation, however we expect that these will

\footnotetext{
${ }^{15}$ We cite here the proceedings of the California Renewable Energy Transmission Initiative, which are in progress. The working data for these proceeding are public, and at the time of writing are available at https://databasin.org/maps/e3616f36144849a9bdc724dc655bc0f9
} 
be mitigated by new market structures in which generation schedules ramp continuously rather than plateau within each hour. Despite accounting for the heavy tails of their distribution, 5 minute forecast errors and 5 minute deviations negligibly impacted the need for load following reserves, even in the most centralized cases which would be most affected by spatial correlation. Future studies that wish to replicate this method may wish to forgo simulating sub-hourly variability to save computation time.

Hourly forecast errors are spatially correlated at long distances, which exacerbates their effect on reserves. However different forecasting methods may be able to reduce this correlation even if they don't reduce forecast errors for each individual system. We imagine some prediction methods may be better at reducing spatial correlation than others, such as the cloud tracking methods described in [40]. An incoming cloud could be predicted with high certainty to cover some fraction of PV plants, though which subset is uncertain. In this case, the forecast for all plants may be poor but the errors in these predictions will be anti-correlated: the subset of panels not covered by the cloud will have been under-predicted, and the subset covered by the cloud will have been over-predicted.

PV systems can provide reserves by curtailing generation in a controlled manner, as noted in recent work $[34,35]$. We find that in all of our scenarios PV can provide between $79 \%$ and $90 \%$ of the additional reserves that result from its inclusion. In the most distributed case, PV would need to curtail less than $5 \%$ of potential energy production. For spatially concentrated scenarios this increases to $15 \%$. We reiterate here that this is a thought experiment to examine the maximum costs of these reserves. In most hours, it will likely be uneconomical for PV to curtail itself to provide reserves because cheaper sources will be available.

Requiring PV systems to smooth their own output results in very large increases in the need for reserves and diminishes the capacity value of solar by as much as $50 \%$. Because most of these reserves are used to manage hourly forecast errors, this result is directly relates to policies that incentivie PV farms to meet their own forecasts. If the penalty for a forecast error is large enough, PV farms may correct their generation to match their forecast rather than the other way around. For example, they could install on site storage to increase or decrease generation, or they could intentionally under-forecast and then curtail any excess generation. Because each of these corrections takes place within individual plants, it will not benefit from any geographic diversity or smoothing.

\section{Acknowledgements}

This work was supported in part by the NSF Graduate Research Fellowship, the California Public Utilities Commission, and NSF grant CNS-1239567. The authors would like to thank E. Carlson and K. Varadarajan of SolarCity, and J. Dise of Clean Power Research, who provided data required for this work.

\section{References}

[1] Jing Wu, Audun Botterud, Andrew Mills, Zhi Zhou, Bri-Mathias Hodge, and Mike Heaney. Integrating solar PV (photovoltaics) in utility system operations: Analytical framework and Arizona case study. Energy, 85:1-9, June 2015. 
[2] Marissa Hummon, Paul Denholm, Jennie Jorgenson, David Palchak, Brendan Kirby, and Ookie Ma. Fundamental Drivers of the Cost and Price of Operating Reserves. Technical report, National Renewable Energy Laboratory (NREL), Golden, CO., 2013.

[3] E. Ela, V. Diakov, E. Ibanez, and M. Heaney. Impacts of variability and uncertainty in solar photovoltaic generation at multiple timescales. Contract, 303:275-3000, 2013.

[4] Debra Lew, Greg Brinkman, E. Ibanez, B. M. Hodge, and J. King. Western wind and solar integration study phase 2. Contract, 303:275-3000, 2013.

[5] G.E. Energy. Western Wind and Solar Integration Study. Technical Report NREL/SR550-47434, National Renewable Energy Laboratory, Golden, CO, May 2010.

[6] David Corbus, J. King, T. Mousseau, R. Zavadil, B. Heath, L. Hecker, J. Lawhorn, D. Osborn, J. Smit, R. Hunt, and others. Eastern wind integration and transmission study. NREL (http://www. nrel. gov/docs/fy09osti/46505. pd f), CP-550-46505, 2010.

[7] Xinggang Bai, Kara Clark, Gary A. Jordan, Nicholas W. Miller, and Richard J. Piwko. Intermittency Analysis Project: Appendix B Impact of Intermittent Generation on Operation of California Power Grid. Technical report, California Energy Commission, 2007.

[8] California Public Utilities Commission. Planning Assumptions \& Scenarios For The 2016 Long Term Procurement Plan Proceeding and The CAISO 201617 Transmission Planning Process. Technical Report R.13-12-010 MP6/avs, California Public Utilities Commission, May 2016.

[9] M. Lave, J. Kleissl, and J.S. Stein. A Wavelet-Based Variability Model (WVM) for Solar PV Power Plants. IEEE Transactions on Sustainable Energy, 4(2):501-509, 2013.

[10] A. Mills and R. Wiser. Implications of Wide-Area Geographic Diversity for Short-Term Variability of Solar Power. Technical report, Lawrence Berkeley National Labratory, 2010.

[11] Richard Perez, Sergey Kivalov, James Schlemmer, Karl Hemker Jr., and Tom Hoff. Parameterization of site-specific short-term irradiance variability. Solar Energy, 85(7):1343-1353, July 2011.

[12] Akinobu Murata, Hiroshi Yamaguchi, and Kenji Otani. A method of estimating the output fluctuation of many photovoltaic power generation systems dispersed in a wide area. Electrical Engineering in Japan, 166(4):9-19, March 2009.

[13] Laura M. Hinkelman. Differences between along-wind and cross-wind solar irradiance variability on small spatial scales. Solar Energy, 88:192-203, February 2013.

[14] Clara M. St Martin, Julie K. Lundquist, and Mark A. Handschy. Variability of interconnected wind plants: correlation length and its dependence on variability time scale. Environmental Research Letters, 10(4):044004, April 2015.

[15] Richard Perez, Sergey Kivalov, Jim Schlemmer, Karl Hemker Jr., and Thomas E. Hoff. Short-term irradiance variability: Preliminary estimation of station pair correlation as a function of distance. Solar Energy, 86(8):2170-2176, August 2012. 
[16] Matthew Lave, Joshua Stein, Jan Kleissl, Abraham Ellis, Clifford Hansen, and Yusuke Miyamoto. Simulating Solar Power Plant Variability for Grid Studies: A Wavelet-based Variability Model (WVM)., October 2011.

[17] Michaelangelo D. Tabone and Duncan S. Callaway. Parameterizing Fluctuations in Solar Photovoltaic Generation Using Hidden Markov Models. In Proceedings of the 2013 IEEE Power \& Energy Society General Meeting, Vancouver, BC, July 2013.

[18] Matthew Lave and Jan Kleissl. Cloud speed impact on solar variability scaling Application to the wavelet variability model. Solar Energy, 91:11-21, May 2013.

[19] J. Wegener, M. Lave, J. Luoma, and J. Kleissl. Temporal downscaling of irradiance data via Hidden Markov Models on Wavelet coefficients: Application to California Solar Initiative data. 2012.

[20] Javier Marcos, Luis Marroyo, Eduardo Lorenzo, David Alvira, and Eloisa Izco. Power output fluctuations in large scale pv plants: One year observations with one second resolution and a derived analytic model. Progress in Photovoltaics: Research and Applications, 19(2):218 227, March 2011.

[21] Javier Marcos, Luis Marroyo, Eduardo Lorenzo, and Miguel Garca. Smoothing of PV power fluctuations by geographical dispersion. Progress in Photovoltaics: Research and Applications, 20(2):226-237, March 2012.

[22] Richard Perez, Thomas Hoff, John Dise, David Chalmers, and Sergey Kivalov. The Cost of Mitigating Short-term PV Output Variability. Energy Procedia, 57:755-762, 2014.

[23] M.D. Tabone and D.S. Callaway. Modeling Variability and Uncertainty of Photovoltaic Generation: A Hidden State Spatial Statistical Approach. IEEE Transactions on Power Systems, 30(6):2965-2973, November 2015.

[24] Mark Rothleder. Track I Direct Testimony Of Mark Rothleder On Behalf Of The California Independent System Operator Corporation, 2011.

[25] H. Holttinen, M. Milligan, E. Ela, N. Menemenlis, J. Dobschinski, B. Rawn, R.J. Bessa, D. Flynn, E. Gomez Lazaro, and N. Detlefsen. Methodologies to determine operating reserves due to increased wind power. In 2013 IEEE Power and Energy Society General Meeting (PES), pages 1-10, July 2013.

[26] M. Milligan, B. Kirby, J. King, and S. Beuning. Operating reserve implication of alternative implementations of an energy imbalance service on wind integration in the Western Interconnection. In 2011 IEEE Power and Energy Society General Meeting, pages 1-8, July 2011.

[27] Energy and Environmental Economics (E3). Investigating a Higher Renewables Portfolio Standard in California. Technical report, January 2014.

[28] Grace C. Wu. Incorporating land-use requirements and environmental constraints in lowcarbon electricity planning for California. Environmental science \& technology, 2014. 
[29] Y.V. Makarov, C. Loutan, Jian Ma, and P. de Mello. Operational Impacts of Wind Generation on California Power Systems. IEEE Transactions on Power Systems, 24(2):1039-1050, May 2009.

[30] Rebecca R. Hernandez, Madison K. Hoffacker, and Christopher B. Field. Land-Use Efficiency of Big Solar. Environmental Science \& Technology, 48(2):1315-1323, January 2014.

[31] O. Bartholomy, T. Vargas, M. Simone, C. Hansen, S. Fitchett, and A. Pohl. Benchmarking solar power and irradiance forecasting accuracy at Sacramento Municipal Utility District. In Photovoltaic Specialist Conference (PVSC), 2014 IEEE 40th, pages 0063-0068, June 2014.

[32] Richard Perez, Sergey Kivalov, James Schlemmer, Karl Hemker Jr., David Renn, and Thomas E. Hoff. Validation of short and medium term operational solar radiation forecasts in the US. Solar Energy, 84(12):2161-2172, December 2010.

[33] C. Goebel and D.S. Callaway. Using ICT-Controlled Plug-in Electric Vehicles to Supply Grid Regulation in California at Different Renewable Integration Levels. IEEE Transactions on Smart Grid, 4(2):729-740, June 2013.

[34] Arne Olson, Ryan A. Jones, Elaine Hart, and Jeremy Hargreaves. Renewable Curtailment as a Power System Flexibility Resource. The Electricity Journal, 27(9):49-61, 2014.

[35] James H. Nelson and Laura M. Wisland. Achieving 50 Percent Renewable Electricity in California. Technical report, Union of Concerned Scientists, Oakland, CA, August 2015.

[36] Lori Bird, Jaquelin Cochran, and Xi Wang. Wind and solar energy curtailment: experience and practices in the United States. National Renewable Energy Laboratory2014, 2014.

[37] California Independent System Operator. California ISO Peak Load History 1998 through 2015. Technical report, 2015.

[38] Thomas E. Hoff and Richard Perez. Quantifying PV power Output Variability. Solar Energy, 84(10):1782-1793, October 2010.

[39] California Energy Commission. Renewable Energy Transmission Initiative 2.0 - Workshops, Notices and Documents, 2016.

[40] Vincent P. A. Lonij, Adria E. Brooks, Alexander D. Cronin, Michael Leuthold, and Kevin Koch. Intra-hour forecasts of solar power production using measurements from a network of irradiance sensors. Solar Energy, 97:58-66, November 2013.

[41] National Renewable Energy Laboratory. Solar Maps. 